\title{
Cascaded Optical Field Enhancement in Composite Plasmonic Nanostructures
}

\author{
V. G. Kravets, ${ }^{1}$ G. Zoriniants, ${ }^{2}$ C. P. Burrows, ${ }^{2}$ F. Schedin, ${ }^{1}$ C. Casiraghi, ${ }^{1,3}$ P. Klar, ${ }^{3}$ A. K. Geim, ${ }^{1}$ \\ W. L. Barnes, ${ }^{2}$ and A. N. Grigorenko ${ }^{1, *}$ \\ ${ }^{1}$ School of Physics and Astronomy, University of Manchester, Manchester, M13 9PL, United Kingdom \\ ${ }^{2}$ School of Physics, University of Exeter, Stocker Road, Exeter, EX4 4QL, United Kingdom \\ ${ }^{3}$ Department of Physics, Free University of Berlin, D-14195 Berlin, Germany \\ (Received 29 July 2010; revised manuscript received 14 October 2010; published 9 December 2010;
} publisher error corrected 14 December 2010)

\begin{abstract}
We present composite plasmonic nanostructures designed to achieve cascaded enhancement of electromagnetic fields at optical frequencies. Our structures were made with the help of electron-beam lithography and comprise a set of metallic nanodisks placed one above another. The optical properties of reproducible arrays of these structures were studied by using scanning confocal Raman spectroscopy. We show that our composite nanostructures robustly demonstrate dramatic enhancement of the Raman signals when compared to those measured from constituent elements.
\end{abstract}

DOI: 10.1103/PhysRevLett.105.246806

The concentration of electromagnetic fields in subwavelength volumes is important in many areas, such as nonlinear optics [1], light-matter interactions [2], photochemistry [3], biophysics [4], laser nuclear fusion [5], optical nanomanipulation [6], biosensing [7], and data storage [8]. Normally, optical fields are concentrated by focusing light with appropriate lenses, the minimum volume of the enhanced field ultimately being determined by the wavelength of the light used. It is well known that metal nanostructures allow one to concentrate light in smaller volumes through the excitation of localized surface plasmons, thereby enhancing the strength of the electric field over that available otherwise [9]. Individual metallic nanoparticles allow a modest field enhancement, of the order of the quality factor $Q$ of the plasmonic resonance [10]. The field strength can be increased further in particle conglomerates $[11,12]$ and particle pairs [13]. The gap between particles is particularly important in many systems [11-14]. Recently, it was suggested that a new class of composite metallic nanostructures might be used to provide very high and well-controlled optical-field enhancements [10].

The confinement of fields using metallic nanostructures, in volumes well below the diffraction limit, down to just several tens of nanometers, involves a process mediated by the electron plasma of the metal [10]. The deeply subwavelength volumes of field confinement and strongly enhanced optical fields that are predicted for composite metallic nanostructures offer many intriguing prospects, for example, 3D optical near-field trapping $[15,16]$. Surface-enhanced Raman scattering (SERS) from single molecules immobilized on "hot" colloidal nanoparticle structures has been reported [17-19] with field enhancements of $\sim 100$ being invoked to account for this extraordinary sensitivity [20]. These results provide an incentive to find ways to produce such high field enhancements in a controlled way, rather than relying on nondeterministic colloidal synthesis techniques.
PACS numbers: 73.20.Mf, 62.23.St, 78.30. $-\mathrm{j}, 78.67 .-\mathrm{n}$

The self-similar plasmonic structure suggested by $\mathrm{Li}$, Stockman, and Bergman [10] presents an appealing design for a composite nanostructure in which the near fields produced by an illuminated large metallic nanoparticle play the role of the exciting field for a smaller metallic nanoparticle, the result of which is to enhance the field further. The electromagnetic field may thus undergo a cascaded enhancement by a factor of up to $g_{\text {tot }} \sim g^{n}$, where $g \sim Q \sim \operatorname{Re} \varepsilon(\omega) / \operatorname{Im} \varepsilon(\omega)$ [10] [ $\varepsilon(\omega)$ is the metal permittivity; e.g., for gold $Q \sim 7$ at a wavelength of $630 \mathrm{~nm}$ ] and $n$ is the number of cascades. Although a self-similar nanoassembly has been demonstrated [21], no experimental verification of any cascaded enhancement of the electromagnetic field by these assemblies was reported. In this Letter, we demonstrate a cascaded enhancement in nanofabricated gold nanostructures of an original design [22], one for which we evaluated a maximal field-enhancement factor of $\sim 100$ for visible light—consistent with our observation of single- or few-molecule SERS.

Our nanostructures comprised three coaxial gold disks of different diameter stacked one on top of another and separated by dielectric spacers. We used the tower-type design which features a large metallic disk of diameter $D$ that has at its center a cylindrical hole of diameter $d$ filled with a dielectric column produced by overexposed poly(methyl methacrylate) (PMMA) [23], a medium-sized metallic disk of diameter $d$ placed on the top of the column, and a small disk of diameter $\delta$ placed on top of that; a schematic and a scanning electron micrograph of the structures studied are shown in Figs. 1(a)-1(c). Owing to the limited resolution of electron-beam lithography, the maximum number of cascades we could achieve with good reproducibility was $n=3$ for a structure with a resonance in the visible. For this work, we chose sizes for the large and medium disks that provided the maximal field enhancement for the excitation wavelength of $\lambda=633 \mathrm{~nm}$ [23], namely, $D \approx 590 \mathrm{~nm}$, $d \approx 110 \mathrm{~nm}$, both of thickness $H=90 \mathrm{~nm}$, and a diameter 
(a)

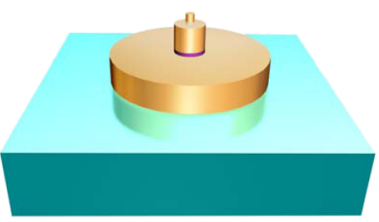

(c)

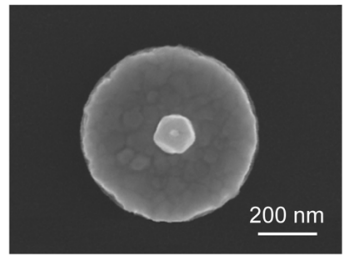

(b)

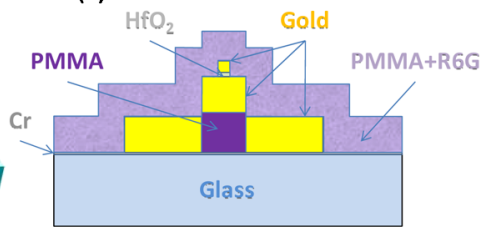

(d)

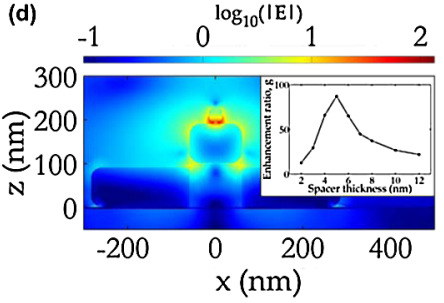

FIG. 1 (color online). Self-similar composite nanostructures. (a) Illustration of the tower-type three-tier structure. (b) Schematic of the cross section of the structure with a coating of PMMA or dye. (c) A scanning electron microscopy image of the structure. (d) Calculated amplitude of the electric field near the structure illuminated by a Gaussian beam in a cross section through the center of the structure. The field magnitudes were normalized to the peak field magnitude of the incident Gaussian beam whose focus was at the center of the smallest disk. The electric field magnitudes are presented on a $\log _{10}$ color scale whose limits are the smallest and largest magnitudes in this cross section. The inset shows the dependence of the maximum of the field enhancement on the thickness of the top dielectric spacer. All field calculations in this work were carried out at $\lambda=633 \mathrm{~nm}$.

$\delta \approx 30 \mathrm{~nm}$ and thickness $h=30 \mathrm{~nm}$ for the small disk. We also fabricated arrays of single large, medium, and small disks for use as references.

Simulations using finite-element modeling [23] of the electromagnetic fields produced by the triple structures in response to an incident Gaussian beam $(\lambda=633 \mathrm{~nm}$, spot size $w=400 \mathrm{~nm}$ ) suggested that the total fieldenhancement ratio $g_{\text {tot }}$ strongly depends on the thickness of the dielectric spacers between the disks. Figure 1(d) shows a typical distribution of the electric field calculated for the experimental geometry used. The inset in Fig. 1(d) shows the sharp dependence of $g_{\text {tot }}$ on the top spacer thickness $s$, with maximal values of $g_{\text {tot }}$ being $>80$. From similar modeling we estimate that for a top spacer thickness of $7 \mathrm{~nm}$ (the experimental value) the maximum field enhancement afforded by (i) the small, medium, and large disks acting on their own to be $7 \pm 1$, (ii) the combination of the middle and lower disks to be $13 \pm 2$, (iii) the combination of the small and middle disks to be $32 \pm 3$, and (iv) the full triple structure to be $44 \pm 4$. Thus, starting from the larger disk, our cascade enhancements are $1.9 \pm 0.5$ and $3.4 \pm 1.0$. In this modeling we averaged the field enhancements over an area of spatial dimension $\sim 1 \mathrm{~nm}$ to avoid numerical noise, and we rounded the corners of the metallic structures to confirm with scanning electron microscopy analysis, subject to a minimum radius of curvature of $5 \mathrm{~nm}$ to avoid spurious high-field points.

To probe the total field enhancement afforded by our triple structures, we used confocal Raman spectroscopy. We coated the nanostructures with a layer of dye molecules, rhodamine 6G (R6G), randomly dispersed in a polymer host, which was a solution of 3\% PMMA in anisole; see Fig. 1(b). The dye concentration in the solution was $10^{-6} \mathrm{M}$, which implies that the average distance between dye molecules in the PMMA film was about $120 \mathrm{~nm}$, and the probability of having one R6G molecule within $\sim 30 \mathrm{~nm}$ of the top disk (i.e., equal to $\delta$ ) was about $20 \%$. The probability of having two R6G molecules within $\sim 30 \mathrm{~nm}$ of the top disk was thus about $4 \%$, indicating that we are in the single molecule regime. We excited the dye by using a confocal laser $(633 \mathrm{~nm})$ arrangement and collected scattered light in the $200-2500 \mathrm{~cm}^{-1}$ band. [As a test, we also measured the response to a green laser $(514.5 \mathrm{~nm})$, for which we did not observe any strong enhancement of either fluorescence or Raman scattering.]

Figure 2 presents the main result of our Letter. Figure 2(a) shows a scanning electron micrograph of two adjacent composite nanostructures separated by $2 \mu \mathrm{m}$ : a triple tower structure (as in Fig. 1) on the left and a double

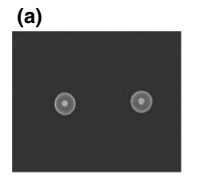

(c)

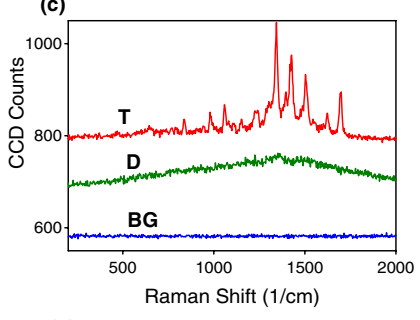

(h)
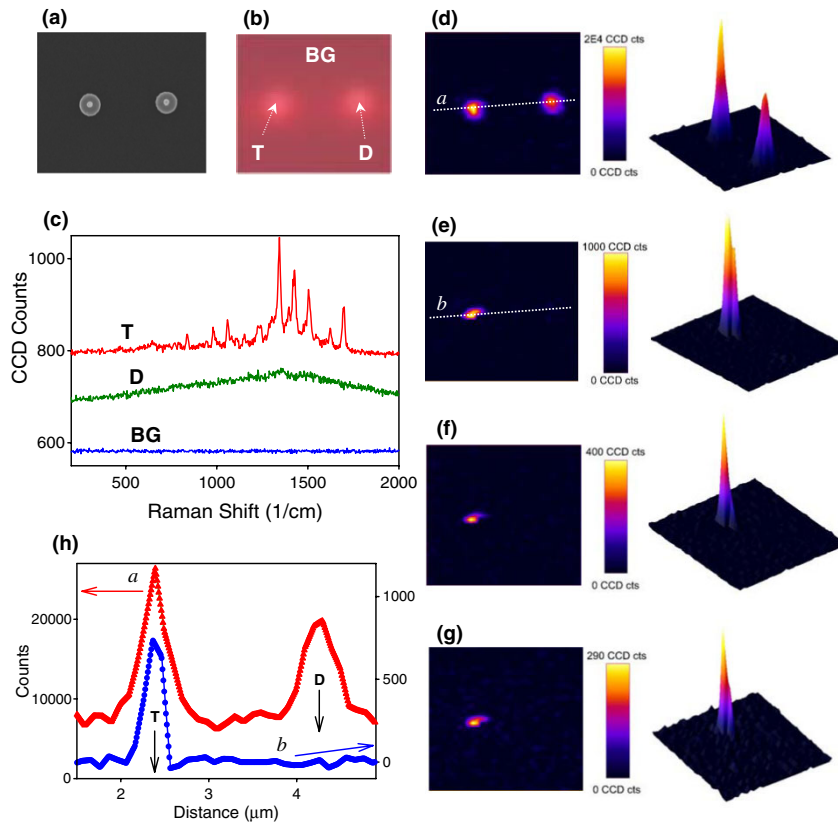

(e)

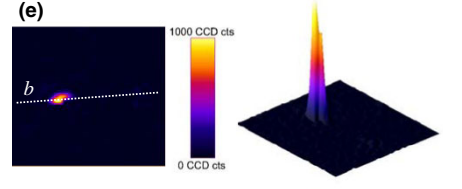

(f)

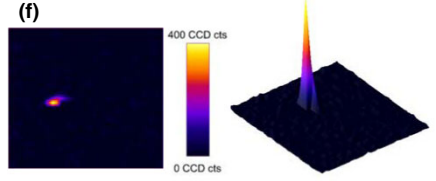

(g)
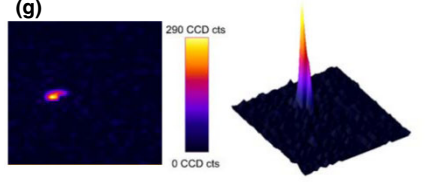

FIG. 2 (color online). The confocal Raman signal from the studied nanostructures at a laser power of $90 \mu \mathrm{W}$ and integration time $50 \mathrm{~ms}$. (a) A scanning electron microscopy image of the triple (left object) and double (right object) structures. (b) An optical image of the triple $(T)$ and double $(D)$ structures. (c) The confocal Raman spectra above the triple structure $(T)$, the double structure $(D)$, and the substrate $(\mathrm{BG})$. The spectra are offset for clarity. (d) An image above the area shown in (a) obtained by integration of the measured spectra in $200-2000 \mathrm{~cm}^{-1}$ range. (e)-(g) Raman images of the studied area at 1350, 1690, and $1230 \mathrm{~cm}^{-1}$, respectively. The color scales have been shifted to zero in order to achieve the best contrast of the images. (h) Line scans along the lines $a$ and $b$ shown in (d) and (e). 
structure (without the top, small, disk) on the right. Figure 2(b) shows the corresponding optical image from these structures in reflected light. We performed Raman mapping by collecting Raman spectra from an array of 50 by 50 points above the whole area shown in Fig. 2(a). The laser power was $P=90 \mu \mathrm{W}$ with a point integration time of $\tau_{\text {int }}=50 \mathrm{~ms}$ (it took about 2 min for the whole scan to complete). Figure 2(c) shows typical Raman spectra measured above the triple structure $(T)$, the double structure $(D)$, and the substrate (BG). The Raman signals measured from single nanoparticles of $\sim 600, \sim 100$, and $\sim 30 \mathrm{~nm}$ were close to the substrate signal (not shown). We see that both $T$ and $D$ traces show enhancement of the Raman background signal, the nature of which is still keenly debated [24]. This enhancement is seen as two bright spots in Fig. 2(d) (and the corresponding inset to the right) obtained by integrating the measured spectra over the frequency range of $200-2000 \mathrm{~cm}^{-1}$ at each scanned point. For brevity, in the rest of the Letter we will refer to this enhanced background as "fluorescence."

The triple structure demonstrates strong enhancement of the Raman signals [seen as well-resolved peaks in the trace $T$, Fig. 2(c)], while the double structure shows no clear sign of Raman peaks, trace $D$ (the Raman signal for this laser intensity and integration time is below the noise level for the double structure). To illustrate this point we have plotted several Raman images by integrating the collected signal at each scanned point for the R6G Raman peaks of 1350 [Fig. 2(e)] and $1690 \mathrm{~cm}^{-1}$ [Fig. 2(f)], as well as for the PMMA peak of $1230 \mathrm{~cm}^{-1}$ [Fig. 2(g)] (the integration interval was $\pm 10 \mathrm{~cm}^{-1}$ for all three images). The Raman images [Figs. 2(e) $-2(\mathrm{~g})]$, and the corresponding insets to the right, clearly demonstrate that only the triple structure yields strong enhancement of the Raman peaks at the studied conditions. We note that the observed Raman signals come from a much smaller area than does the fluorescence. To check the size of the area around the nanostructures in which the fluorescence and Raman signals are enhanced, we plotted line scans along lines $a$ [Fig. 2(d)] and $b$ [Fig. 2(e)] in Fig. 2(h). We see that the fluorescence signal (generated by both the triple and the double structures) has a spatial extent (half-width) of $\sim 0.6 \mu \mathrm{m}$, while the Raman signal (seen only for the triple structure) has a spatial extent of $\sim 0.3 \mu \mathrm{m}$. The results shown in Fig. 2 were typical for arrays of double and triple structures. We found that about $10 \%$ of the triple structures showed large Raman signals (from a total sample of about 700). This percentage correlates well with the probability of finding an R6G molecule in the direct vicinity of the top particle in the cascade and suggests that the cascaded enhancement is a robust phenomenon.

Having established that the triple structures yield much stronger Raman signals for both R6G and PMMA molecules than the double (and single) structures, we turn our attention to a quantitative evaluation of the total field enhancement. Although the theory of SERS is a fascinating subject discussed in detail in several excellent reviews and recent publications [25-29], the use of SERS to estimate the local electromagnetic field enhancement faces several difficulties. First, there is a consensus that, in addition to the well-documented electromagnetic enhancement mechanism, SERS can be also enhanced due to other mechanisms such as ground-state chemical interactions, dynamic charge-transfer, etc. [30]. Second, the process of Raman scattering leaves a molecule in an excited state which has then to relax to the ground state, usually via nonradiative processes (phonons), and is random in nature. In the case where one or only several molecules contribute to SERS, different channels compete with each other, which leads to nonreproducible Raman signals [31]. Third, if the field enhancement near a nanostructure is sufficiently high, other nonlinear processes may drive a Raman active molecule into a metastable state, effectively switching it off which could result in intermittency (blinking) [20,31-33] or lead to coherent effects in Raman scattering (peak splitting, etc.) [34]. In summary, enhancements evaluated from Raman measurements are a guide rather than an exact measure of the electromagnetic enhancmenets offered by nanostructures.

We illustrate the difficulties connected with the evaluation of local field enhancements from Raman signals in Fig. 3. Figure 3(a) shows a temporal set of Raman spectra measured above a triple structure $\left(P=88 \mu \mathrm{W}, \tau_{\text {int }}=1 \mathrm{~s}\right.$, $w \sim 500 \mathrm{~nm}$, and the spectra are offset for clarity). One can see that the Raman spectra vary with time, different peaks taking prominence in different scans. For an increased $P=200 \mu \mathrm{W}$ and a longer $\tau_{\text {int }}=60 \mathrm{~s}$, we
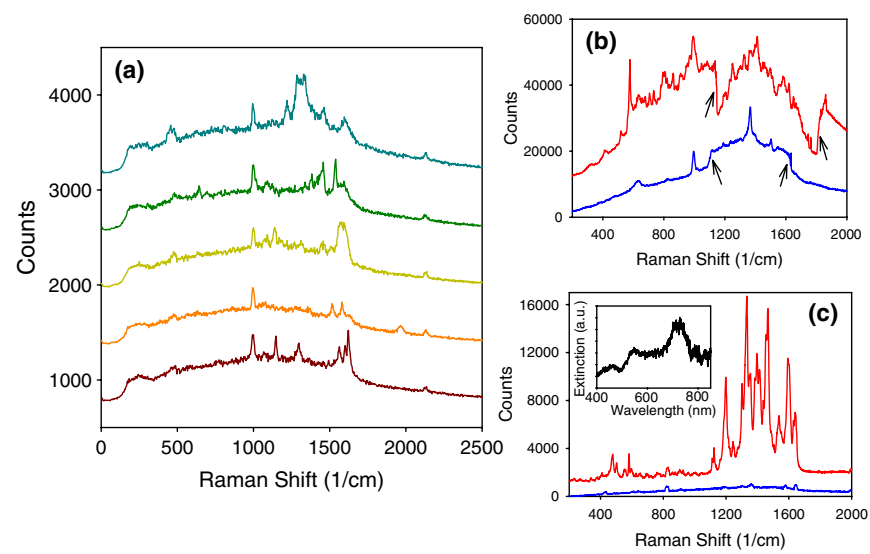

FIG. 3 (color online). The Raman signal from the studied nanostructures. (a) A temporal set of the Raman spectra measured at $P=88 \mu \mathrm{W}$ and $\tau_{\text {int }}=1 \mathrm{~s}$; the spectra are offset for clarity. (b) The Raman spectra measured at $P=200 \mu \mathrm{W}$ and $\tau_{\text {int }}=60 \mathrm{~s}$. (c) The Raman spectra from the triple structure (the red curve) and the double structure (the blue curve) measured at $20 \mu \mathrm{W}$ and $\tau_{\text {int }}=60 \mathrm{~s}$. The inset shows the extinction spectrum of the composite structure with a subtracted contribution from the chrome underlayer. 
recorded well-defined jumps in the measured spectra for triple structures denoted by arrows in Fig. 3(b). The jumps may correspond to a molecule switching on and off during recording of the spectra. This indicates that the bulk of the signal in our case is produced by just a few R6G molecules. We found that the most stable Raman spectra were obtained with low laser powers (which alleviated the problem of intermittency) and longer integration times (which allowed us to achieve time averaging of the Raman signals instead of spatial averaging); cf. [31]. Figure 3(c) shows the Raman spectra measured at $P=$ $20 \mu \mathrm{W}$ and $\tau_{\text {int }}=60 \mathrm{~s}$ above the triple structures (the red curve) and double substrates (the blue curve).

By comparing the signals from the triple and double nanostructures, we estimate that the ratio of the field enhancement observed for triple and double structures (the cascaded enhancement factor) is $\sim 3.6 \pm 0.2$ for $\lambda=633 \mathrm{~nm}$. This estimate is based on the root of 4 th power from the ratio of Raman peaks [35] where we have chosen weak and medium R6G peaks of $1460,1300 \mathrm{~cm}^{-1}$, etc., in order to avoid bulk contribution from the background (data not shown). It is probably a conservative lower limit of the enhancement factor, since it does not account for the greater number of R6G molecules that contribute to the double structure signal due to the larger size of the top disk of the double structure $(110 \mathrm{~nm})$ compared to the top disk of the triple structure $(30 \mathrm{~nm})$. Our estimated field-enhancement factor between double and triple structures compares well with the factor of $3.4 \pm 1.0$ determined from finite-element modeling. By comparing fluorescence from single and double structures, we previously evaluated the field enhancement for double structures of the studied size as $30 \pm 7$; for details, see [23]. Consequently, we can estimate the field-enhancement ratio for the optimal triple structures reported here as $g_{\text {tot }} \sim 100$.

In summary, we have demonstrated reproducible cascaded optical-field enhancement from well-controlled regular arrays of three-tier composite plasmonic nanostructures produced by nanofabrication. The field enhancement was probed by using a thin layer of dye molecules. We observed significant Raman signals above three-tier composite plasmonic nanostructures even at lower laser intensities $(10 \mu \mathrm{W}$, spot size $\sim 500 \mathrm{~nm})$ and short integration times (20-50 ms). Finite-element modeling provides broad quantitative support for our experimental findings. We hope that these results will add impetus to the exploration and exploitation of more complex metallic nanostructures as a means to enhance optical fields at the nanoscale in a controllable way.

V. G. K. and A. N. G. acknowledge the support of a FP7 METACHEM grant. W. L. B. acknowledges the support of the Royal Society. C.P. B. was supported in part by GreatWesternResearch and Qinetiq. C.C. and P. K. acknowledge support from the Alexander von Humboldt Foundation.
*Corresponding author.

sasha@manchester.ac.uk

[1] S. Kim et al., Nature (London) 453, 757 (2008).

[2] T. H. Taminiau et al., Nat. Photon. 2, 234 (2008).

[3] K. Watanabe et al., Chem. Rev. 106, 4301 (2006).

[4] X. Michalet et al., Science 307, 538 (2005).

[5] K. W. D. Ledingham, P. McKenna, and R. P. Singhal, Science 300, 1107 (2003).

[6] D. G. Grier, Nature (London) 424, 810 (2003).

[7] B. Rothenhausler and W. Knoll, Nature (London) 332, 615 (1988).

[8] W. A. Challenger et al., Nat. Photon. 3, 220 (2009).

[9] S. Kuhn et al., Phys. Rev. Lett. 97, 017402 (2006).

[10] K. Li, M. I. Stockman, and D. J. Bergman, Phys. Rev. Lett. 91, 227402 (2003).

[11] F. J. García-Vidal and J. B. Pendry, Phys. Rev. Lett. 77, 1163 (1996)

[12] A. M. Michaels, J. Jiang, and L. Brus, J. Phys. Chem. B 104, 11965 (2000); J. P. Camden et al., J. Am. Chem. Soc. 130, 12616 (2008).

[13] P. Muhlschlegel et al., Science 308, 1607 (2005).

[14] A. N. Grigorenko et al., Nature (London) 438, 335 (2005).

[15] M. Righini et al., Nature Phys. 3, 477 (2007).

[16] A. N. Grigorenko et al., Nat. Photon. 2, 365 (2008).

[17] K. Kneipp et al., Phys. Rev. Lett. 78, 1667 (1997).

[18] S. Nie and S. R. Emory, Science 275, 1102 (1997).

[19] J. A. Dieringer, R. B. Lettan, K. A. Scheidt, and R. P. Van Duyne, J. Am. Chem. Soc. 129, 16249 (2007).

[20] E. C. Le Rue et al., J. Phys. Chem. C 111, 13794 (2007).

[21] S. Bidault, F. J. Garcia de Abajo, and A. Polman, J. Am. Chem. Soc. 130, 2750 (2008).

[22] Self-similar nanolenses of a lateral design, as in [10], fabricated with the help of electron beam lithography did not show a cascaded field enhancement probably due to the presence of a glass substrate.

[23] V. G. Kravets et al., Nano Lett. 10, 874 (2010).

[24] S. Mahajan et al., J. Phys. Chem. C 114, 7242 (2010).

[25] M. Moscovits, Rev. Mod. Phys. 57, 783 (1985).

[26] A. Otto, I. Mrozek, H. Grabhorn, and W. Akemann, J. Phys. Condens. Matter 4, 1143 (1992).

[27] K. Kneipp et al., J. Phys. Condens. Matter 14, R597 (2002).

[28] E. C. Le Ru, E. Blackie, M. Meyer, and P. G. Etchegoin, J. Phys. Chem. C 111, 13794 (2007).

[29] L. G. Cancado et al., Phys. Rev. Lett. 103, 186101 (2009).

[30] L. Jensen, C. M. Aikens, and G. C. Schatz, Chem. Soc. Rev. 37, 1061 (2008).

[31] D. K. Lim, K.-S. Jeon, H. M. Kim, J.-M. Nam, and Y.D. Suh, Nature Mater. 9, 60 (2009).

[32] J. Jaing et al., J. Phys. Chem. B 107, 9964 (2003).

[33] E. C. Le Ru, E. C. Meyer, and P. G. Etchegoin, J. Phys. Chem. B 110, 1944 (2006).

[34] V. M. Akulin and N. V. Karlov, Intense Resonant Interactions in Quantum Electronics (Springer, Berlin, 1992), Lecture 13.

[35] E. C. Le Ru and P. G. Etchegoin, Chem. Phys. Lett. 423, 63 (2006). 